\title{
Role of optical flow field asymmetry in the perception of heading during linear motion
}

\author{
LAURA TELFORD and IAN P. HOWARD \\ York University, North York, Omtario, Canada
}

\begin{abstract}
During linear translation through a stationary environment, the pattern of optical flow generated on each retina is symmetrical when the head is aligned with the heading, but during lateral gaze the optical flow is asymmetric. We assessed whether human subjects could use the magnitude of this asymmetry to determine the direction of heading during passive translation through a 3-D environment. When allowed to move their heads in order to look in the direction of self-motion, subjects indicated their heading precisely and accurately. When the head was locked in alignment with the misaligned body, and gaze adjustments were not allowed, responses were quite precise, but showed a large bias which increased with increasing heading angle.
\end{abstract}

Linear self-motion through a stationary environment creates a radial pattern of optical flow on the retina. When the angle of gaze is fixed, the motion path is specified by the center of expansion in the flow field (Gibson, 1950). However, when the eyes move, a linear component is superimposed on the radial flow pattern. Several methods have been proposed to decompose retinal flow into components due to linear translation and eye rotation (reviewed in Warren \& Hannon, 1990). The essential task is that of detecting the common linear component resulting from eye rotations and subtracting it from the total pattern, leaving only the radial optical flow due to linear self-motion. A copy of the motor command signal to the eyes could be used to specify the linear component (Sperry, 1950; von Holst, 1954). Royden, Banks, and Crowell (1992) have shown heading judgments to be less accurate when pursuit eye movements are simulated by flow field manipulations than when the eyes actually move, suggesting that eye position signals are taken into account prior to heading judgments. The linear flow component due to eye rotation may also be specified visually because eye rotation produces a flow pattern that does not depend on the distance of stimulus elements from the eye. In contrast, the direction of each radial flow vector (for a smooth surface) is determined by its meridional direction from the locus of heading, and the magnitude of each vector is determined by its distance from the locus of heading and from the eye (Nakayama \& Loomis, 1974).

This research was carried out at the Centre for Vision Research at York University and was supported by a grant from the Natural Sciences and Engineering Research Council of Canada. We would like to thank Masao Ohmi for software development and Jeff Laurence for help in design and construction of the apparatus. Correspondence concerning this article may be addressed to I. P. Howard, Institute for Space and Terrestrial Science, Room 103 Farquharson Building, York University, North York, ON, Canada M3J 1P3, or to L. Telford, Box 605, Department of Neurology, University of Rochester, 601 Elmwood Ave., Rochester, NY 14642 (e-mail: telford@cvs.rochester.edu)
Decomposition of retinal flow into components due to translation and rotation may not be necessary if the observer is moving through a 3-D scene, since the differential motion parallax occurring between elements at different distances from the observer can be used to specify heading (see Cutting, 1986). To the left of the heading, the images of nearer objects move to the left relative to those of more distant objects. To the right of the heading, the images of nearer objects move to the right relative to those of more distant objects. The images of objects that are collinear with the motion path remain visually aligned. This locus of zero parallax can therefore specify heading direction, and this cue to heading is not affected by eye movements (see Longuet-Higgins \& Pradzny, 1980, for a more detailed discussion). During binocular vision, each eye has a distinct locus of zero parallax for objects at near distances. For a homogeneous scene, when both eyes look in the direction of heading, the flow pattern in each eye is symmetrical and the two flow patterns form a bilaterally symmetrical pair. If the direction of gaze is not parallel with the direction of heading, the flow pattern is still symmetrical about the locus of zero parallax, but more of the flow field is seen on one side of the visual field than on the other. The degree of this visual field asymmetry provides information about the magnitude of the gaze deviation that can be used to center the gaze on the locus of zero parallax. This notion that asymmetries in the optical flow field can be used to guide a series of fixations toward the heading has previously been proposed, but not tested, by Whitman Richards (1975).

When gaze is so eccentric that the locus of zero parallax is no longer in view, it is theoretically possible to use the motion parallax gradient to extrapolate the locus of zero parallax to provide an estimate of the headcentric direction of self-motion. Warren (1976) examined this possibility by using computer-generated visual displays that simulated self-motion relative to a ground plane. Subjects were asked to align an unseen pointer with the heading, which was either inside or outside of the field of view. Point- 
ing errors were larger for intermediate headings outside the field of view $\left(30^{\circ}-75^{\circ}\right)$ than for headings within the field of view $\left(0^{\circ}\right.$ and $\left.15^{\circ}\right)$, but when the heading was $90^{\circ}$ to the left of the gaze position, pointing errors were the same as those obtained in the $15^{\circ}$ condition. Warren suggested that some heading angles may be preferentially encoded by the visual system. However, performance was less than perfect even at the preferred heading angles. Constant errors in the "movie" condition, which simulated a view of the ground plane from a moving vehicle, were less than a degree in the straight-ahead condition, but for all other heading angles, the error was more than $3^{\circ}$. This relatively poor performance for the eccentric conditions may not reflect limitations of the visual system, but may be due to the fact that the 2-D displays contained no motion parallax cues. Cutting (1986) has shown that such cues can greatly facilitate heading judgments. He used computergenerated visual displays simulating observer approach toward three planar surfaces separated in depth. Simulated depth between the displays and gaze angle relative to the motion path were manipulated, and subjects were asked to report whether the displays simulated leftward, rightward, or straight-ahead trajectories. When there was no depth between the surfaces, performance was near chance, but improved as the separation between the surfaces increased.

We measured the ability of human observers to judge their heading while moving through a 3-D environment. Heading judgments made when subjects were allowed to move their heads to align the locus of zero parallax with the fovea were compared with those made when the head was held at a fixed angle relative to the heading. In the first case, subjects could use the asymmetry of the flow field to guide a series of fixations toward the heading and align the locus of zero parallax with the fovea. However, when the head was fixed, the locus of zero parallax was imaged in the retinal periphery and the degree of flow asymmetry provided the most salient information about the direction of heading.

A secondary consideration in this experiment was whether vestibular stimulation could facilitate judgments when the locus of zero parallax was not imaged on the fovea. Since the peripheral retina lacks the high resolution of the fovea, it is possible that knowledge of the position of the head relative to the motion axis derived from the vestibular system could improve the accuracy and precision of judgments of the direction of self-motion.

\section{METHOD}

\section{Subjects}

Five subjects ( 3 females, 2 males; mean age, 32 years) with corrected-to-normal vision participated in this experiment. Subjects requiring optical correction wore contact lenses. None of the subjects had any known visual or vestibular deficits.

\section{Apparatus}

Passive visual or visual-vestibular stimulation was provided by accelerating subjects in a cart at sub- or supravestibular values along a track inside a hallway lined with mirrors $(6.0 \mathrm{~m}$ long, $1.4 \mathrm{~m}$ wide).
Subjects sat on a seat that was padded to reduce vibration. The seat could be rotated on a base until the subject was disoriented and then locked into one of eight orientations relative to the motion path $\left( \pm 5^{\circ}\right.$, $\pm 10^{\circ}, \pm 15^{\circ}, \pm 20^{\circ}$ ). The base, which had wheels, could be pulled along a rail on the floor of the hallway by a cable driven by a DC servomotor. The motor servo system (EG\&G Torque Systems) was controlled by a Macintosh SE computer.

Visual stimulation was provided by a helmet-mounted optical system. A rectangular frame housed two $15-\mathrm{cm}$ monitors (Panasonic WV-5200B; resolution, 600 lines) in front of the subject's eyes and supported two $5.5-\mathrm{cm}$ video cameras (Panasonic; focal length, $7.5 \mathrm{~mm}$ ) on staiks $38 \mathrm{~cm}$ above the subject's head. The cameras were positioned in the same frontal plane as the axis of head rotation and were separated laterally by roughly the human interpupilary distance $(6.7 \mathrm{~cm})$. The frame was pivoted on a central shaft, which was aligned with the vertical rotation axis of the head. The optical assembly was carried on two vertical columns attached to the sides of the cart. Subjects wore a fiberglass helmet with individually fitted molded foam inserts so that any sideways head rotation carried the entire optical system with it. The optical system could be rotated $\pm 20^{\circ}$ and locked into position with a magnetic clutch. During linear cart motion, subjects binocularly viewed a $60^{\circ}$ wide optical flow field on the monitors, which was generated as the cameras translated through a field of 360 white vertical rods ( $36 \mathrm{~cm}$ long, $0.5 \mathrm{~cm}$ wide) suspended from the ceiling of the hallway. Since the walls of the hallway were lined with mirrors, subjects saw a stereoscopic display of vertical rods extending indefinitely in all directions. The monitors were viewed through lenses which magnified the image and accommodated the eyes at infinity. Rubber flanges eliminated extraneous light.

\section{Sensory Stimulation Conditions}

All subjects received three trials in each of the sensory stimulation conditions for each heading angle. The chair was oscillated very slowly in the dark for approximately $40 \mathrm{sec}$ and then was locked into position at one of the eight heading angles. During oscillation, the head was locked in alignment with the body. Prior to linear acceleration, the head was either unlocked (head-free conditions) or maintained in the same head-fixed position. Head-fixed and head-free conditions were run in which subjects either viewed the optic flow field on the two monitors (visual conditions) while fusing two small black fixation points $\left(0.2^{\circ}\right)$ attached to the front of the monitors, or simply fused the spots against a stationary gray field on the monitors (no-vision conditions). In all four conditions, subjects were accelerated at subthreshold values $(0.0006 \mathrm{~g})$ for $33 \mathrm{sec}$ to a peak velocity of $0.2 \mathrm{~m} / \mathrm{sec}$ and were then decelerated to zero velocity over another $33 \mathrm{sec}$. Head-fixed and head-free conditions were also run at suprathreshold accelerations to provide combined visual-vestibular stimulation. In these conditions, subjects were accelerated at $0.05 \mathrm{~g}$ to a peak velocity of $2.8 \mathrm{~m} / \mathrm{sec}$ over $5.3 \mathrm{sec}$ and were decelerated to zero over a further $5.3 \mathrm{sec}$. Because the cart moved faster during the visual-vestibular trials, subjects had less time to judge their heading direction. However, we compared results obtained in the visual headfree condition with those in a similar condition run in another experiment (Telford, Howard, \& Ohmi, 1995) in which visual judgments were made in the same amount of time as that available in the visualvestibular condition and determined that performance was not significantly affected by the amount of time available.

Optical flow rates were determined by the observer's velocity and by the distances of each of the individual display elements (rods) from the observer. At the start of each trial, the farthest rods were $6 \mathrm{~m}$ from the observer, but distances became very small $(<1 \mathrm{~cm})$ as the cameras moved past the rods.

In all conditions, subjects aligned an unseen Plexiglas rod, which was located at hand level on a support attached to the front of the chair, in the direction of motion. A potentiometer attached to the shaft of the pointer recorded pointer position. The pointer and potentiometer were precalibrated with reference to a protractor positioned below the pointer. Analogue outputs from the potentiometer 
were converted to a digital representation of pointer position, which was linear over the range used.

Subjects with no previous experience in this apparatus were given approximately eight practice trials with feedback; experienced subjects were given three practice trials with feedback.

\section{RESULTS}

At the end of each trial, digital readouts from the pointer potentiometer were recorded. Pointing accuracy (constant error) was calculated as the mean of the signed deviations of each of the settings from the true heading. Pointing overshoots were assigned positive values, and pointing undershoots were assigned negative values. The standard deviation of the three settings for each heading provided an index of pointing precision (variable error). Precision and accuracy data were each subjected to repeated measures analysis of variance (ANOVA) with conditions and heading angle as main effects. When a significant $F$ was obtained, Tukey HSD tests were performed to test pairwise significance.

Pointing precision is shown for each of the six stimulus conditions in Figure 1 as a function of heading angle. Leftward and rightward heading angles did not differ significantly and have been combined. Responses in the two nonvisual conditions were much more variable than those in the other conditions. The ANOVA was significant for the main effect of conditions $[F(5,22)=5.43, p<.005]$. Only comparisons between each of the visual conditions (headfixed and head-free visual and visual-vestibular) and the two nonvisual conditions (head-fixed and head-free) were significant. Pointing precision did not vary as a function of heading angle.
Constant pointing errors are shown in Figure 2 as a function of heading angle. Leftward and rightward pointing responses have been collapsed together, since both directions resulted in errors of the same sign and magnitude. The main effect of conditions was highly significant $[F(5,22)=16.30, p<.0001]$. All of the visual and visualvestibular conditions were significantly different from the two nonvisual conditions $(p<.05)$. When visual information was available, subjects overestimated (positive errors) their heading angle for both leftward and rightward headings, but when vision was not available, subjects underestimated (negative errors) their heading angle for leftward and rightward headings. The two head-fixed conditions in which visual information was available (visual and visual-vestibular) were also significantly different from the corresponding head-free conditions $(p<.01)$. The main effect of heading angle was not significant, but there was a significant conditions $\times$ heading angle interaction $[F(35,154)=3.18, p<.0001]$. The two head-fixed conditions in which vision was available (visual and visualvestibular) were significantly different $(p<.05)$ from all other conditions for heading angles larger than $5^{\circ}$. At the $15^{\circ}$ heading angle, the two nonvisual conditions were significantly different from the other four conditions.

\section{DISCUSSION}

We examined whether human observers could use the degree of asymmetry in the optical flow field to make accurate and precise heading judgments in the absence of gaze adjustments. Our findings indicate that while subjects could accurately and precisely point in the direction of their motion when visual information was available and

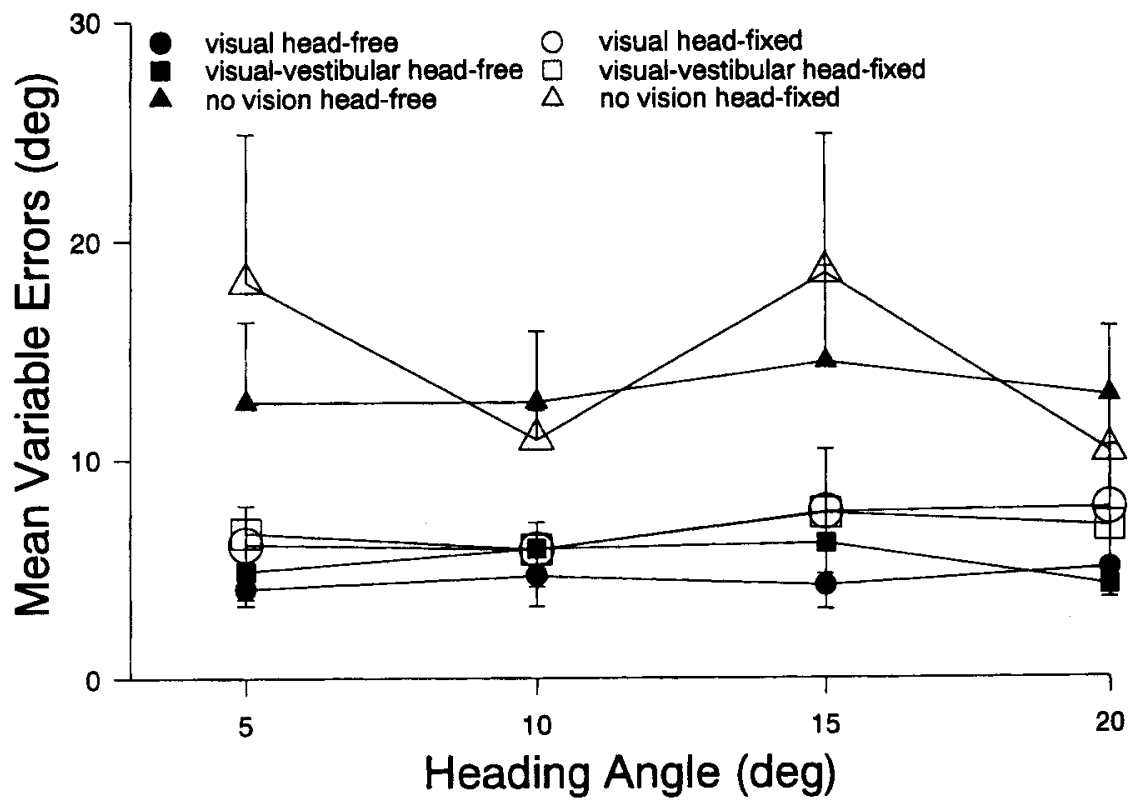

Figure 1. Mean variable errors as a function of heading angle $(\boldsymbol{n}=5)$. Leftward and rightward headings did not differ significantly and have been combined. Conditions in which subjects were not free to move their heads are shown with open symbols; the head-free conditions are represented by filled symbols. Error bars represent standard errors of the mean. 


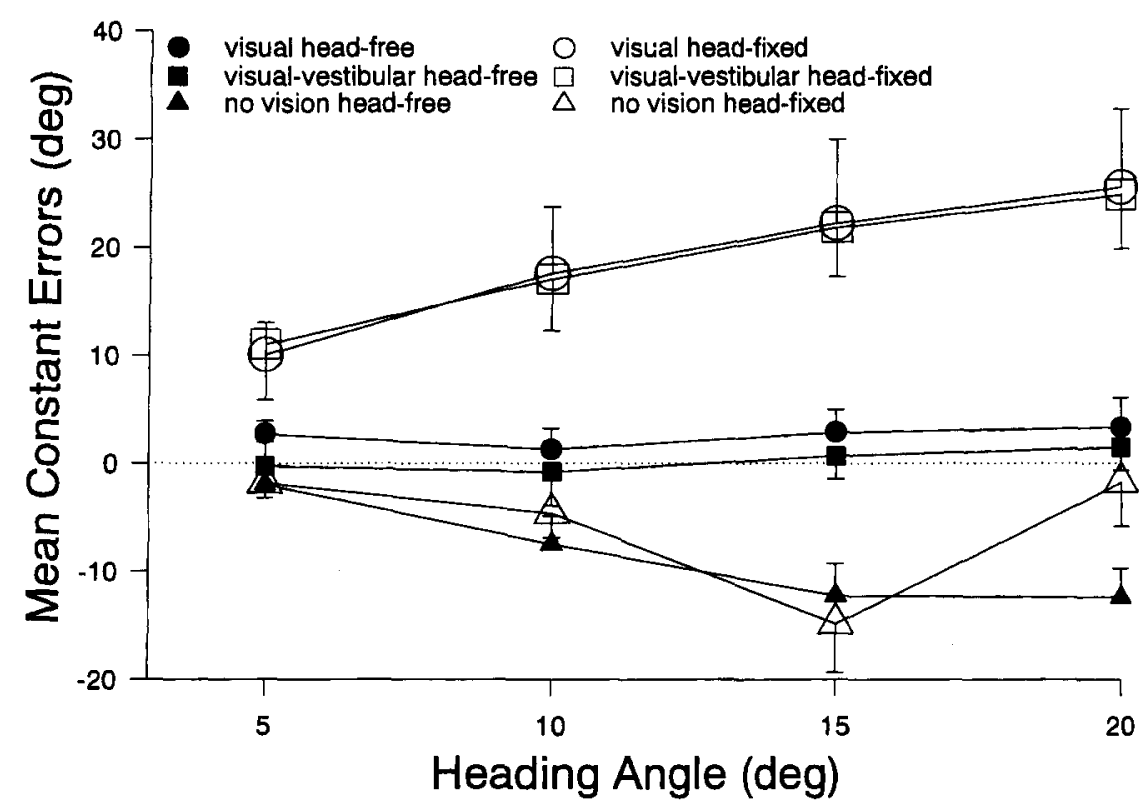

Figure 2. Mean constant errors as a function of heading angle $(n=5)$. Leftward and rightward headings did not differ significantly and have been combined. Open symbols show head-fixed conditions; closed symbols show head-free conditions. Error bars represent standard errors of the mean.

the head was free to move, large constant pointing errors $\left(>25^{\circ}\right.$ at the most extreme heading) away from the center of outflow were made when head movements were restricted. Pointing precision was unaffected, indicating that subjects responded with a high degree of confidence. Thus, it appears that when the locus of zero parallax is imaged in the retinal periphery, subjects cannot use the degree of flow field asymmetry, in the absence of gaze adjustments, to guide accurate heading judgments.

Nonvisual factors such as adaptation of sensory receptors in the neck cannot be responsible for the less accurate pointing responses in the head-fixed conditions, since the errors obtained in the visual and nonvisual head-fixed conditions were in opposite directions. Subjects underestimated their heading angle when visual cues were absent and overestimated their heading when visual cues were available. In any case, adaptation is unlikely to occur when the head is in its primary position, in alignment with the body. Adaptation should only occur if the head is in an eccentric position relative to the neck (Howard \& Anstis, 1974). It is, however, possible that the motor feedback resulting from active head rotation in the head-free conditions could have improved performance. Findings from a study by Royden et al. (1992) suggest that motor feedback from the eyes can provide information that facilitates heading judgments. It is therefore possible that motor signals generated during head rotation could also provide heading information.

We cannot be certain which features of the optical flow field subjects used to guide heading judgments in the visual head-free conditions. Since the heading was within the field of view in all visual conditions, both the degree of flow field asymmetry and the position of the locus of zero parallax on the retina could have been used to compute the heading. The degree of flow field asymmetry was probably the more salient of the two cues for guiding the head from the initial eccentric gaze position toward the heading, because its identification does not require high resolution. Since the falloff in response accuracy in the head-fixed conditions parallels the decline in receptor resolution from the fovea to the retinal periphery (Howard, 1982), subjects may not have been able to identify the locus of zero parallax to determine heading when it was imaged in the retinal periphery. However, in the head-free conditions, the locus of zero parallax could be brought into a region of high retinal acuity and aligned with the central fixation spot.

The optical flow fields generated during the head-fixed linear motion trials in this experiment contained at least two sources of asymmetry. In the eccentric gaze conditions, the velocity field was asymmetrical and each visual field was stimulated asymmetrically by horizontal motion in a particular direction. Although in this experiment, the two possibilities could not be distinguished, work by Dyre and Andersen $(1992,1994)$, in which the trajectories of individual image elements and their velocities were dissociated, suggests that velocity gradients play a significant role in heading perception.

Another potential source of visual information available in the visual head-free, but not head-fixed, conditions is the motion parallax resulting from head rotation. Some motion parallax is generated in the optical flow field as a result of head rotation because the eyes are located in front of the axis of rotation. However, in this experiment, head rotation produced minimal parallax, since the sensors, 
which in this case were the cameras, were in the same plane as the axis of head rotation. In any case, any parallax resulting from head rotation would have produced a constant shift in the optical flow in the direction of the head rotation, generating larger, not smaller, constant errors.

Although most studies which have simulated a fixed gaze angle relative to the heading have reported a consistent heading bias (Crowell \& Banks, 1993; Cutting, 1986; Cutting, Springer, Braren, \& Johnson, 1992; Warren \& Kurtz, 1992), the bias obtained in the present investigation was much larger. Generally, in previous studies, computergenerated optical flow fields have been viewed by stationary observers, a situation quite unlike that reported here, in which mobile observers traversed a stationary 3-D world. Computer-generated displays that have simulated motion through clouds of random dots consist of radially expanding flow fields that contain both vertical and horizontal motion near the center of outflow. Since our display was composed of vertical rods that were viewed through an aperture, no vertical motion was present. If the head is fixed at a small angle relative to the heading, a flow field containing only horizontal flow may appear to be more asymmetrical than one containing both vertical and horizontal flow, thereby generating a larger response bias. However, at large gaze angles, the optical flow in radial patterns will also appear to be quite asymmetrical, since the flow becomes more and more lamellar, until only horizontal flow is seen at gaze angles near $90^{\circ}$.

We have previously shown that pointing in the direction of heading with an unseen pointer is not an optimal test of visual heading perception (Telford et al., 1995). Subjects generate larger errors when aligning an unseen pointer with the heading than they do when aligning the head with the direction of motion. Aligning the eyes with the heading involves identifying the locus of zero parallax in the flow field and moving the eyes to minimize image slip on the retina. If the eyes fixate a head-fixed visual target, the task reduces to moving the head to minimize retinal slip. Aligning an unseen rod with the heading requires supplemental information from other senses that provide information concerning head-on-body, arm-on-body, and bodyin-space relations. Thus, in the absence of other sensory information, subjects would be expected to generate larger errors when performing a rod-pointing than a head or an eye-pointing task. However, inadequacies of the rodpointing task cannot explain the $10^{\circ}-25^{\circ}$ errors observed in the head-fixed visual and visual-vestibular conditions but not in the same head-free conditions, since the same task was used in all conditions.

When gaze was fixed relative to the motion path, our subjects tended to overestimate their heading angle. The direction of this bias corresponds to that reported by Warren (1976). However, Johnston, White, and Cumming (1973) and Llewellyn (1971) have reported constant errors in the opposite direction. Warren (1976) noted that this difference could be due to the fact that these studies simulated approach toward a single planar surface. Relative motion occurring between objects at different distances from the observer may provide an important source of heading information. In the absence of relative depth information, subjects may be forced to use a variety of different strategies to find the locus of heading. Johnston et al. (1973) noted that some subjects initially fixated the screen center and then followed a dot as it moved away from the center, at which point they would pick up a new dot near the heading. Since dot velocities near the locus of outflow were lower, subjects tended to underestimate the heading, making estimates between the true heading and the screen center. In the present investigation, subjects were instructed to fixate a point in the screen center and were not allowed to follow individual flow vectors to find the heading. The large flow asymmetry associated with large heading angles may have biased estimates in the direction of the flow - that is, away from the screen center.

We examined whether concordant vestibular stimulation provided additional information to enhance heading judgments during head-free or head-fixed visual stimulation. Neither precision nor accuracy was influenced in either condition. It is not surprising that the addition of vestibular cues did not facilitate visual performance in the headfree condition, since vision alone was enough to produce highly precise and accurate pointing responses. However, in the head-fixed condition, in which vision alone produced a strong response bias away from the true heading, the otoliths should have provided veridical information about the direction of head motion. If information about the direction of head acceleration was put together with proprioceptive information from the neck, subjects should have been able to point in the direction of self-motion in the absence of vision. However, we have previously shown (Telford et al., 1995) that heading estimates based solely on otolithic information are both imprecise and inaccurate. It is therefore quite conceivable that in the absence of other sensory inputs, the brain relies heavily on visual rather than vestibular information to guide heading.

\section{REFERENCES}

Crowell, J. A., \& Banks, M. S. (1993). Perceiving heading with different retinal regions and types of optic flow. Perception \& Psychophysics, 53, 325-337.

Cutting, J. E. (1986). Perception with an eye for motion. Cambridge, MA: MIT Press.

Cutting, J. E., Springer, K., Braren, P. A., \& Johnson, S. H. (1992) Wayfinding on foot from information in retinal, not optical, flow. Journal of Experimental Psychology: General, 121, 41-72.

Dyre, B. P., \& ANDERSEN, G. J. (1992). Are trajectory-based models of heading going in the wrong direction? Investigative Ophthalmology \& Visual Science, 33, 1141.

Dyre, B. P., \& Andersen, G. J. (1994). Statistical moments of retinal flow may be used to determine heading. Investigative Ophthalmology \& Visual Science, 35, 1269.

GiBson, J. J. (1950). Perception of the visual world. Boston: Houghton Mifflin.

HOWARD, I. P. (1982). Human visual orientation. London: Wiley.

Howard, I. P., \& ANSTIS, T. (1974). Muscular and joint-receptor components in postural resistance. Journal of Experimental Psychology, 103, 167-170.

Johnston, I. R., White, G. R., \& Cumming, R. W. (1973). The role of optical expansion patterns in locomotor control. American Journal of Psychology, 86, 31 1-324.

LLEWELLYN, K. R. (1971). Visual guidance of locomotion. Journal of Experimental Psychology, 91, 245-261. 
Longuet-Higgins, H. C., \& Prazdny, K. (1980). The interpretation of a moving retinal image. Proceedings of the Royal Society of London: Series $B, \mathbf{2 0 8}, 385-397$.

NaKaYAma, K., \& LoOMis, J. M. (1974). Optical velocity patterns, velocity sensitive neurons, and space perception: A hypothesis. Perception, 3, 63-80.

RichARDS, W. (1975). Visual space perception. In E. C. Carterette \& M. P. Friedman (Eds.), Handbook of perception (Vol. 5, pp. 351-386). New York: Academic Press.

RoYden, C. S., BANKs, M. S., \& Crowell, J. A. (1992). The perception of heading during eye movements. Nature, 360, 583-585.

SPERRY, R. W. (1950). Neural basis of the spontaneous optokinetic response produced by visual inversion. Journal of Comparative \& Physiological Psychology, 43, 482-489.

Telford, L., Howard, I. P., \& OHMI, M. (1995). Heading judgments during active and passive self-motion. Experimental Brain Research, 104, 502-510.

vON HoLST, E. (1954). Relations between the central nervous system and the peripheral organs. Animal Behavior, 2, 89-94.

WARREN, R. (1976). The perception of egomotion. Journal of Experimental Psychology: Human Perception \& Performance, 2, 448456.

WARREN, W. H., \& HANNON, D. J. (1990). Eye movements and optical flow. Journal of the Optical Society of America, 7, 160-169.

WARREN, W. H., \& KURTZ, K. J. (1992). The role of central and peripheral vision in perceiving the direction of self-motion. Perception \& Psychophysics, 51, 443-454.

(Manuscript received July 8, 1994;

revision accepted for publication July 23,1995 .) 